\title{
Implications of Inertia for Assessing Drug Control Policy: Why Upstream Interventions May Not Receive Due Credit
}

Short Title: Implications of Inertia

\author{
Jonathan P. Caulkins \\ Carnegie Mellon University \\ Qatar Campus and H. John Heinz III School of Public Policy \& Management \\ 5000 Forbes Ave. \\ Pittsburgh, PA 15213 \\ caulkins@cmu.edu \\ 1-412-268-9590 (office) \\ $1-412-268-5338$ (FAX)
}

December, 2007

\section{Acknowledgments}

This work was funded in part by the Robert Wood Johnson Foundation, the Qatar Foundation, and the Colonial Foundation through its support of Australia's Drug Policy Modeling Program. Alison Ritter and Paul Dietze collaborated in the development of the drug use model that forms a foundation for this research. 


\title{
Implications of Inertia for Assessing Drug Control Policy: Why Upstream Interventions May Not Receive Due Credit
}

\begin{abstract}
Background: There is ongoing interest in assessing the effectiveness of various drug control strategies, including policy intended to reduce initiation and prevalence.

Compartmental models of trajectories of drug use have been developed that demonstrate that drug "systems” display significant inertia; interventions on systems with high inertia can be difficult to evaluate.
\end{abstract}

Method: The implications of inertia are illustrated by combining a new empiricallyderived model of national drug initiation with a compartment model of trends in illicit drug use parameterized for Australia.

Results: Typical initiation trajectories seem to display damped oscillation. Lags and interpersonal variation in the trajectories of drug use careers smooth those oscillations for measures of problem use and total drug-related social cost. Even abrupt changes in initiation - as dramatic as a two-year long 50\% reduction in initiation - barely ripple the trajectory of drug-related social cost. The benefits of upstream interventions are not diminished but rather are hidden in plain sight by being spread broadly over time so that they leave only the faintest finger-print detectable by before-and-after comparison - even if the intervention has a large effect and a perfect counter-factual is available.

Conclusion: Fluctuating initiation is not inconsistent with ever worsening drug problems, and the absence of clear drops in drug-related problems does not imply that an upstream intervention did not produce substantial social benefits. Hence, macro-level evaluation of upstream drug control interventions has inherent limitations. Drug use and related problems are often perceived of as stubborn, even intractable. Some of that perception stems from real limitations in the effectiveness of drug-control interventions. However, inertia inherent in the evolution of drug use can make even cost-effective interventions appear weak (and detrimental interventions appear benign).

(Keywords: Dynamic modeling, drugs, epidemic, compartmental models, costeffectiveness, evaluation) 


\section{Introduction}

Two empirical regularities concerning illicit drug use seem hard to reconcile. Initiation ebbs and flows over time, sometimes dramatically, but drug problems tend only to get worse or at best stabilize. These observations appear inconsistent, but to the extent that they can both hold for the same substance, they seem to suggest that interventions designed to reduce initiation and overall prevalence are of limited value. Both those inferences can, however, be exactly wrong.

Drug use at the individual and societal levels progresses through stages (Everingham et al., 1995; Kandel, 2002). Many individuals start by using only cannabis. Some quit; others progress to other substances. A few who progress go on to problematic use (e.g., injection or dependent use). At the societal level, new drugs can pass through phases of exponential growth in initiation, stabilization at endemic levels, and sometimes subsequent decline. Systems models, also known as “compartment” or "stocks and flows” models are used to describe these staged patterns of use, particularly for heroin (Rossi, 2001, 2004), cocaine (Homer, 1993; Everingham et al. 1995; Caulkins et al., 2004), and tobacco (Mendez and Warner, 2000; Levy et al., 2002; Levy and Friend, 2002).

Systems models can reconcile the mystery of fluctuating initiation and steadily worsening drug problems and show that their coexistence in no way implies that upstream interventions are not cost-effective. The mechanisms are not novel for systems theorists. There are even terms of art (aka jargon) for describing them, but such jargon obscures more than it illuminates, so this paper illustrates the concept with numerical examples grounded in real data.

The model of how individuals stochastically progress through stages of use comes from a recent compartmental model of drug use in Australia (Caulkins et al., 2007). The trajectory of initiation over time is a new composite based on US data for ten different substances. We are not trying to model any single drug or country. Rather, the point is to illustrate how general characteristics of drug use combine to create the apparent paradox (fluctuating initiation coinciding with ever worsening problems) and the temptation to be unduly pessimistic concerning the value of upstream drug use control interventions. 
The next section describes the drug use and initiation models. The subsequent section uses numerical simulations to illustrate the key points. The final section discusses implications for drug control evaluation and policy.

\section{Data and Methods}

\subsection{Model of Drug Use Trajectories}

We use a discrete-time, annual-step compartmental model of illicit drug use with constant annual transition probabilities that was developed for the Australian Drug Policy Modeling Program (DPMP) (Caulkins et al., 2007; Ritter et al., 2007). The model has states representing five types of drug use. Initiation is into one of two non-injecting states, one representing the use only of cannabis (denoted $C$ ), the other (state $M$ ) reflecting non-injection use of other substances (and possibly cannabis as well). From these states, people may quit or escalate into any of three injecting drug use states. One (state $H$ ) reflects frequent or "heavy" injection drug use. The other two both represent less frequent ("light”) injection drug use, one each for people who will and who will not eventually escalate to frequent injection ( $L_{e}$ and $L_{o}$, respectively). (That distinction was driven by data availability. Infrequent injection by people who will eventually escalate can be studied retrospectively via samples of dependent injectors. Parameters for infrequent injectors who never escalate had to be estimated in other ways.)

Figure 1 shows the five states along with all possible transitions. Escalation from cannabis-only directly to frequent injection is possible, though it occurs at a lower rate than does either escalation from cannabis-only to other non-injection use or escalation from other non-injection use to injection use. The model was parameterized using data from general population surveys, data from heroin users who were resuscitated by ambulance paramedics, and other efforts to model injection drug use in Australia. The parameterized model fit well historical patterns of drug use up through the onset of the so-called Australian heroin drought (Day et al., 2004; Degenhardt et al., 2005). (The model does not track supply or availability, so it cannot be expected to reproduce the effects of supply shocks on patterns of use.) With constant initiation the system approaches a unique, stable steady state. 
Figure 1. Diagram of Drug Use Model’s Five States and Associated Transitions

The model can be expressed concisely in mathematical terms as:

$\left[\begin{array}{l}C(t+1) \\ M(t+1) \\ L_{e}(t+1) \\ L_{o}(t+1) \\ H(t+1)\end{array}\right]=\left[\begin{array}{ccccc}0.8328 & 0.08 & & & \\ 0.0650 & 0.8350 & & 0.05 & \\ 0.0020 & 0.008 & 0.7810 & & \\ 0.0080 & 0.0320 & & 0.85 & \\ 0.0009 & 0.0036 & 0.2190 & & 0.95\end{array}\right]\left[\begin{array}{c}C(t) \\ M(t) \\ L_{e}(t) \\ L_{o}(t) \\ H(t)\end{array}\right]+\left[\begin{array}{c}0.9112 \\ 0.0888 \\ 0 \\ 0 \\ 0\end{array}\right] I(t)$

where

$C(t)=$ number of users of cannabis only in year $t$,

$M(t)=$ number of non-injection users of other drugs in year $t$,

$L_{e}(t)=$ number of occasional IDUs in year $t$ who will escalate to regular use,

$L_{o}(t)=$ number of occasional IDUs in year $t$ who will not escalate to regular use,

$H(t)=$ number of regular injection users in year $t$, and

$I(t)=$ number of initiates in year $t$.

Caulkins et al. (2007) supplement this prevalence model with rough estimates of average social costs per person-year spent in each model state based on Moore's (2006) estimates of social cost by substance. The resulting estimate for frequent injection ( $H$ state) is far higher (point estimate of $\$ 66,572$ per person-year) than for infrequent injection (both $L$ states, $\$ 1,967$ ), general non-injection use ( $M$ state, $\$ 1,967$ ), or use of cannabis only ( $C$ state, \$750). The number of digits to which these estimates are reported should not be construed as an indication of precision. The estimates are very rough, but their key characteristic is to embody a belief that the final, most problematic drug use state accounts for perhaps $70 \%$ of all social costs.

\subsection{Trajectories of Initiation}


We seek an initiation model that is typical for a range of substances. The general pattern is apparent from inspection of various data sets: rapid rise followed by an ebbing and then sometimes a second wave. However, we need a specific quantitative description.

Data for this were drawn from the US Household Survey because its unrivaled sample sizes allows year-by-year estimation even for less common drugs. The number of people initiating by year since 1960 was estimated for ten substances using each of the Household Surveys from 1999 through 2005 via the variables IRsubYFU, where "sub" is an abbreviation for the name of the substance. (Total sample size 388,617.) The separate estimates were averaged (simple average since surveys were of similar sizes) and scaled as a proportion of the peak level of initiation before 1990. (That peak is usually the alltime peak, but hallucinogens and stimulants had much later "second waves" of initiation that eventually exceeded the initial peak.) Replication with data from other countries would be interesting and is an appealing avenue for further work.

Different substances penetrate the market at different times, so it is best to measure time relative to a particular point in the "epidemic cycle" not relative to a specific calendar year. The timing of peaks can be ambiguous. Some drugs immediately reach their overall peak. Others plateau and then fluctuate, sometimes slightly exceeding the initial peak in the next few years. Hence, it is preferable to align the periods of fastest growth rather than the peaks. This requires shifting the initiation curves for cocaine and crack back substantially (7 and 15 years, respectively), and the curves for PCP (3 years), heroin, methamphetamine, and sedatives (1 year each) more modestly.

Figure 2 shows the resulting initiation curves over time. It is impossible to distinguish visually the individual curves for all ten drugs, but that is not a problem since the overall point of the graph is that there is considerable consistency across these ten quite different substances. The lines for three of the four major drugs are drawn in bold so they can be identified (solid line for marijuana, dashed for methamphetamine, and dotted for cocaine). The line for the fourth major (heroin) is more variable, due to smaller numbers of respondents having used it. Bolding it makes the overall graph harder to read, but it is in the middle of the pack except in the first ten years post-peak, 
when it had a sharper than average decline in initiation post-peak. The two drugs that stand out for having initiation fall the most and longest are PCP and sedatives.

Figure 2: US Drug Initiation Over Time As a Percentage of the Pre-1990s Peak; Time Scaled Shifted to Align the Periods of Rapid Growth in Initiation

The general pattern observed in Figure 2 is increase to a peak, ebbing, rebound to a lower peak, and then (for drugs whose time series extend this far), another downturn. In short, US initiation to date might be said to exhibit damped oscillation. Beyond the first two years, this damped oscillation can be represented by the function ${ }^{1}$

$$
I(t)=A\left(1-e^{-\lambda t} \cos (\omega t)\right)
$$

where

$$
\begin{aligned}
& \lambda=\text { rate of damping of oscillations } \\
& \omega=\text { frequency of oscillations } \\
& A=\text { amplitude. }
\end{aligned}
$$

Figure 3 juxtaposes Figure 2's marijuana initiation curve and the average of the curves for the other nine drugs alongside this oscillatory function with parameters corresponding to an oscillation period of 26.5 years $(\omega=2 \pi / 26.5)$, damping with a halflife of about 14 years $(\lambda=0.05)$, and an amplitude $A=2 / 3$. The amplitude governs the long-run level. The other parameters make the peak nearly 1 (1.018 to three digits), so in the long-run initiation stabilizes at about two-thirds of its peak level.

Figure 3: A Simple Model of Damped Oscillation Plotted Alongside Historical Trends in US Initiation of Marijuana and the Average of Nine Other Drugs, Shifted to Align the

$$
\text { Periods of Rapid Increase in Initiation }
$$

\section{Results}

\footnotetext{
1 With an oscillator, what goes up must have been doing down in the recent past, but historically the initial sharp rise in initiation comes after periods of very little initiation, not a time when initiation was crashing. Hence, the fit cannot be extrapolated backwards in time. In particular, the fit is good from year three on, but cannot be extrapolated back to cover the first two years for which we have data (1960 and 1961 for a drug such as marijuana).
} 
We combine the models of initiation and stochastic escalation in drug use to explore implications for aggregate or population-level trajectories of drug use and related problems. All of the figures below include four lines: initiation, overall (total) prevalence of past-year drug use, prevalence of injection drug use, and drug-related social cost. For readability, the four lines are scaled so they do not overlap and always appear in that order when read from bottom to top. Because of the scaling and because the focus is on trends not absolute levels, the vertical axis is omitted.

The first question is, what does the typical trajectory of a drug problem look like? One might expect that if initiation oscillates as in Figure 3 then drug use and drug-related problems necessarily would as well, but Figure 4 shows that is not the case. Feeding the oscillating initiation function described above into the compartmental model of drug use produces modest fluctuations in overall drug use prevalence but steady growth in drugrelated social costs.

Figure 4. Damped Oscillation in Initiation Produces Modest Fluctuations in Prevalence and Steady Growth in Drug-Related Social Cost

Of course if the oscillations in the initiation "forcing function" are great enough, then they will percolate through the model and affect even the later injection drug use states, so one might wonder whether the surprising result in Figure 4 stems from unrealistically rapid damping (technically, a $\lambda$ parameter that is too large). Figure 5 shows that even in the extreme case where $\lambda$ is set to 0 (no damping), so initiation forever bounces back and forth between 0 and its maximum value, the resulting fluctuations in the social-cost estimate are still modest ( $7.5 \%$ from peak to trough). The reason is that the lags involved in escalation from first use to heavy use and eventual quitting from heavy use are both long (approaching the same duration as the period of oscillation in initiation, or $\sim 25$ years), and variable (meaning some people escalate and/or desist more quickly than others do).

Figure 5. Even Un-Damped Oscillation in Initiation Produces Only Modest Fluctuations in Drug-Related Social Cost 
Such strong oscillation in initiation creates sizable oscillations in drug prevalence because overall past-year prevalence is dominated by the first two drug use states (cannabis only and non-injection use of other substances). Note also the model lags are visible in the succession of peaks. Initiation peaks first. Overall prevalence peaks about five years later; injection drug use and drug-related social cost peak still five years after that.

The previous two figures show that it is perfectly possible for drug problems to worsen steadily even as initiation ebbs and flows. Indeed that is not only possible; it is to be expected. It follows rather mechanically from the basic structures of drug use.

The resolution of this apparent empirical paradox has a crucially important corollary for drug policy. If the consequences of natural fluctuations in initiation are invisible to someone looking at downstream indicators of drug-related consequences, then so too will be the benefits of policy-induced reductions in initiation. The remainder of this section is devoted to elaborating this fundamental point about absence of readily visible success not necessarily implying failure for upstream interventions.

Before proceeding, four points are in order. First, the invisibility applies not only to total drug-related social cost, but also to any indicator that is driven primarily by the behavior of the most problematic stages of use at the end of the drug use escalation chain. Second, the numerical simulations pertain to reductions in initiation, but the implications extend beyond prevention programming both because other interventions might affect initiation (e.g., supply shocks, cf. Weatherburn et al., 2003; Day et al., 2006) and because although the lags and smoothing are greatest when tweaking initiation, they also exist for fluctuations in rates of escalation and for quitting from the early model states. The broad point is best understood as applying to any program that affects the general prevalence of use in the early (non-injection) drug use states, not just to prevention programs. Third, the invisibility applies to fluctuations in either direction; the harm of programs or events that increase rather than decrease general prevalence will likewise be hard to detect when looking only at downstream indicators. Fourth, the model is entirely linear with no endogenous feedback, so the principle of supposition implies that every initiate generates the same expected social cost. Consequently, every initiation that is prevented produces 
the same expected value. For example, cutting initiation by $50 \%$ reduces the social costs for that initiation cohort by $50 \%$. In this model invisibility of effects has nothing to do with intervening at a stage in the epidemic cycle when the intervention is less costeffective than it might have been if employed earlier or later.

The invisibility of reductions in initiation to people focused on downstream indicators stems from two distinct mechanisms. We illustrate each in turn, with two different numerical simulations. The first mechanism is that drug use problems take a long time to approach their steady state levels, so most of our experience comes from countries dealing with the growth stage of an epidemic. When the overall problem is growing, a successful intervention may merely reduce the rate of growth and not create any reduction in a problem indicator. Before and after comparisons might underappreciate the value of policy interventions during that growth stage because strong counterfactuals are usually lacking for indicators at the societal level.

The second mechanism is that even interventions whose effects on initiation are focused in time will create benefit streams that are spread over many decades. That diffusion in onset of effects makes it hard to detect a successful intervention's signal in an impulse-response sense, even when a perfect counter-factual is available.

To illustrate the first mechanism we consider the extreme example of a policy that instantaneously and permanently cuts all drug initiation by $50 \%$, starting the year after initiation peaks. (Timing the effect to hit on the downside of initiation's oscillatory cycle amplifies the relative decline in initiation, so this is "best case" with respect to seeing an effect and hence conservative with respect to our main point.) Quite literally, this means using the same initiation trajectory as in Figure 4 except that in year 13 and every year thereafter, initiation is exactly half what it was in Figure 4. Figure 6 shows the resulting trajectories of initiation, overall prevalence, prevalence of injection, and social cost. The key point is that the magnitude of the drug problem (as reflected in drug-related social costs) grows steadily and even smoothly in the face of this massively successful policy intervention.

Figure 6. Effects of a Permanent 50\% Reduction in Initiation Starting in Year 13 and Relative to the Figure 4 Base Case 
There are two ways to get a sense of how long one would have to wait until this first mechanism ceases to be important. First, Figure 4 shows the social cost curve can still be growing steadily 70 years after the onset of rapid growth in initiation. Second, Caulkins et al. (2007) estimate that if pre-drought conditions had persisted in Australia, long-run injection drug use would have grown to twice its 2000 levels. Again, this stems from long average delays between initiation and escalation to regular injection. The average is long even though this model includes pathways that allow for rapid escalation. Furthermore, Australia has a relatively large culture of injection without dependence. So the Australian parameterization might have made those rapid-escalation pathways more prominent than they would be in some other countries.

In the previous figure, one reason the reduction in initiation did not produce a reversal of the upward trend in social costs was that costs were still going up in delayed response to the growth in initiation over the previous years. That is, the delayed response to over a decade of increasing initiation masked the benefits of the reduction in initiation. In the jargon of evaluation studies, a before-and-after study (looking for a downturn in social costs) would fail because the past is not the correct counterfactual for the future. The decline in initiation made social costs go up less quickly than they otherwise would have, but it did not make them go down.

One great advantage of working with simulation models is that the counterfactual trends are readily available. One merely has to run the model with the baseline initiation. Doing so reveals the second distinct but equally serious problem for evaluating upstream interventions at the aggregate level, namely that the benefits of interrupting initiation get spread out over time.

This can be appreciated intuitively by thinking about the effects of successful intervention on one person who would otherwise have escalated to a long career of dependent use. If that person would have begun problematic use in 10 years and persisted in that use for 15 years, the bulk of the benefits of prevention would be spread over the period from 10 to 25 years in the future. No single year is likely to exhibit more than one-fifteenth of the total benefit. Furthermore, for fourteen of the fifteen years for which there is a substantial benefit, that benefit would be no larger than in the preceding year. 
The only time when a simple before and after comparison would detect much benefit is when comparing the $11^{\text {th }}$ year to the $10^{\text {th }}$.

In reality, it is even harder than this to detect year-to-year changes because escalation is not a step function. Intensity of use and hence many social harms may grow more smoothly even for one individual. Furthermore, not everyone waits exactly ten years before escalating to problematic use. Some would have escalated after eight. Others after twelve. So at the population level, escalation in the amount of problem use averted by successful prevention is even less abrupt.

The clearest way to illustrate this concept is to start the model in steady state with constant initiation, so the counterfactual is complete stability in all indicators. We then "shock" the system with a dramatic reduction in initiation. Any deviations in the resulting indicators from complete stability can be attributed to the shock. Figure 7 does this for a two-year long 50\% reduction in initiation beginning in year 10 . In the long-run, this will avert exactly $100 \%$ of a full year's worth of drug-related social costs. Yet Figure 7 shows it is not easy to discern its effects on problem use, let alone social costs, even with a perfect counterfactual. The largest reduction in social cost relative to the counterfactual is just 2.2\% (occurring 19 years after the reduction in initiation). The largest year-on-year reduction is less than $0.5 \%$.

Figure 7. Halving Initiation for Two Years Creates Only a Modest Ripple in Future Drug-Related Social Costs, Even When a Perfect Counter-Factual Is Available

\section{Discussion}

The main finding is that lags and inertia in realistic models of drug use smooth out the consequences of fluctuations in initiation. Even large and abrupt changes in initiation do not have impacts that can easily be detected in population level indicators of problem drug use, harm indicators, or social cost.

This is similar to but a stronger version of the distinction between incidence (flow into a population) and prevalence (stock of that population). The flow vs. stock distinction can be captured in a one-state model that is often explained intuitively by 
comparison to a bathtub with a partially open drain. ${ }^{2}$ Incidence is like the flow of water into the tub from the faucet. Prevalence is the amount of water in the tub. Turning the faucet (initiation) off for a minute does not immediately empty the tub (eliminate use). More generally, the pool of water (existing users) creates inertia in the system's response to changes in faucet settings (prevention programs), and it would be hard to tell what had been happening to the inflow just by measuring the depth of the water (prevalence), particularly if the water was choppy (there is random measurement error in surveys or other measures of drug use).

The main result here is that drug systems are like this, but much worse. A more precise metaphor would be a chain of several interconnected tubs. Initiation flows primarily into the first tub. Flow into the other tubs comes primarily from flow out of the preceding tub in the chain. Indicators of harm or social cost are driven by the most problematic drug users, and their population is represented by the last tub in the chain. Monitoring the level in that last tub (which incidentally has the choppiest water/most measurement error) is an all but futile way to assess whether someone has temporarily turned down the faucet feeding the first tub in the chain. Downstream monitoring is similarly ineffective at assessing other upstream interventions (e.g., attempts to bail water out of the first tub), so the basic point applies not only to drug prevention but also to upstream interventions (e.g., creating a temporary shortage of supply that drives uncommitted users out of the market).

There are multiple implications of the fact that it is hard to evaluate upstream interventions by looking at downstream indicators. One purely descriptive implication is reconciling two superficially contradictory empirical regularities: (1) initiation ebbs and flows but (2) drug problems continually worsen. We observe that the overall initiation pattern for ten drugs in the US over the last 45 years might best be described as damped sinusoidal oscillation. However, feeding that initiation profile into our compartmental model of drug use shows that even such pronounced fluctuations in initiation can have little discernable effect on downstream measures of societal-level problem use or drug-

\footnotetext{
2 If the drain is partially open, then leaving the faucet on indefinitely fills the tub to the point where the outflow through the drain just offsets the inflow from the faucet. If the drain were wide open, the tub might never fill appreciably. If it were closed, the tub would overflow.
} 
related social costs. Furthermore, the drug system takes so long to fill tubs at the end of the chain that indicators of problem use can still be increasing 50 or more years after initiation is first turned on. Hence, fluctuations in initiation are in no way inconsistent with steadily worsening drug problems, particularly in the first five decades of a drug problem.

An implication for policy is that upstream interventions should not be expected to produce visible effects on societal-level indicators of drug-related harm or social cost. Consider the scenario from Figure 7 - a two-year long 50\% reduction in initiation relative to steady state levels. Few programs produce such reductions even in the shortrun, so this shock to initiation is large. Yet even with a perfect counter-factual it does not produce reductions in annual drug-related social cost that were abrupt (largest year to year drop was just $0.5 \%$ ) or deep (maximum reduction of 2.2\%).

It is crucial to understand that this near-invisibility of effects absolutely does not mean reductions in initiation are not valuable. The model above is linear and has no feedback. So the principle of supposition ensures it is a mathematical inevitability that cutting initiation by $50 \%$ for two years will, over time, eliminate exactly one year's worth of steady state social cost. Since drug problems are still growing, annual costs in steady state presumably exceed contemporary estimates of the annual social cost of illicit drug use, figures that even now are on the order of \$6B for Australia and \$180B for the United States (Collins and Lapsley, 2002; Harwood et al., 1998).

We are not saying that prevention's effects are nearly-invisible because they do not exist. Whether prevention is effective or not is an empirical matter not addressed by this paper. Rather, we are saying that even if there were a prevention program that was spectacularly effective - saving the equivalent of \$6B in Australia or \$180B in the US that program's effects would be nearly invisible to people focused on downstream indicators. So the absence of an effect on downstream indicators cannot be construed as evidence against the effectiveness of upstream interventions.

This warning clearly pertains to casual attempts to draw inferences on the fly from eyeballing trends in aggregate indicators, but there are also limitations to even sophisticated time series analysis. The impulse response function for downstream indicators is very smooth; dealing with that requires a lot more than including a half- 
dozen lagged variables. The maximum response to a $50 \%$ cut in initiation was just $2.2 \%$. For a more realistic change in initiation (say 10\%), the magnitude of the "signal" would be smaller $(<1 \%)$ and the random variation in population level measures of heavy use can be quite large. Furthermore, an evaluation of plausible duration would under-appreciate the delayed effects of upstream interventions. In Figure 7, adding up all benefits for a full decade after the end of the interruption in initiation would still only capture $14 \%$ of the benefits of that interruption.

Consider what this implies for the ability of empirical evaluations to inform strategic drug policy choices. Imagine policy makers said: "Drug policy is so important we are willing to give the scientific community ten full years to empirically evaluate our new policy before we make any decisions." Scientists would have to answer, "Ten years is not enough.” The policy makers could be forgiven for concluding, "Then you are useless, because with study periods any longer than that, the drug problem and the technology of drug control will have changed so much that your evaluations will be out of date before the ink is dry on the report."

This is not a broadside against all evaluations. Evaluations that use systems models to project longer-term consequences of effects observed to date can transcend this limitation, and interventions targeted directly at problem drug users do not have to wait long to see whether they produce important benefits. Also, one can still evaluate prevention programs' effects on proxy variables - such as self-reported prevalence threeyears post-intervention. What is futile is trying to evaluate upstream interventions by looking at trends in downstream indicators such as emergency room mentions, fatal overdoses, etc.

Note, the dispersion of downstream effects to the point of invisibility works both ways. Counter-productive interventions that increase initiation or overall prevalence in ways that greatly increase drug-related social costs can do so without leaving fingerprints. So the fact that a policy change does not produce any detectable change in problem drug use trends does not prove its innocence.

The meta-point is that one cannot evaluate broad national drug control interventions the way we evaluate simple programmatic interventions with a before and after mindset looking at a few years, or even a five- or ten-year, time horizon. 
What can be done? Ideally the scientific community would create systems models that allow policy simulations of the sort above but with a precision that makes them relevant to particular policy choices, not merely illustrative of general limitations of empirical evaluations. Given limitations on both data systems and basic understanding of drug "systems dynamics", that is a long-run research endeavor.

In the mean time, the main practical implication is a warning to avoid the temptation to draw the wrong conclusions. The model offers an analytical defense of studious agnosticism when it comes to drawing inferences about the long-run effects of policies that could affect drug initiation or other flows shaping overall drug prevalence.

\section{References}

Caulkins, J.P., D.A. Behrens, C. Knoll, G. Tragler, and D. Zuba (2004). Markov chain modeling of initiation and demand: the case of the U.S. cocaine epidemic. Health Care Management Science, 7, 319-329.

Caulkins, J.P., P. Dietze, \& A. Ritter (2007) Dynamic Compartmental Model of Trends in Australian Drug Use. Healthcare Management Science. 10(2), 151-162.

Caulkins, J.P., R. Pacula, S. Paddock, \& J. Chiesa (2002). School-Based Drug Prevention: What Kind of Drug Use Does it Prevent? Santa Monica, CA: RAND.

Caulkins, J.P., C.P. Rydell, S.S. Everingham, J. Chiesa, \& S. Bushway (1999). An Ounce of Prevention, a Pound of Uncertainty: The Cost-Effectiveness of School-Based Drug Prevention Program. Santa Monica, CA: RAND.

Collins DJ, Lapsley HM (2002). Counting the Cost: Estimates of the social costs of drug abuse in 1998-99, Canberra: Commonwealth of Australia.

Day, C., L. Degenhardt, S. Gilmour, and W. Hall (2004). Effects of reduction in heroin supply on injecting drug use: Analysis of data from needle and syringe programmes. British Medical Journal, 329, 428-429.

Day, C., L. Degenhardt, and W. Hall (2006). Changes in the initiation of heroin use after a reduction in heroin supply. Drug and Alcohol Review, 25, 307-313.

Degenhardt, L., P. Reuter, L. Collins, and W. Hall (2005). Evaluating explanations of the Australian 'heroin shortage'. Addiction, 100, 459-469. 
Everingham, S.S., P. Rydell, and J.P. Caulkins (1995). Cocaine consumption in the US: Estimating past trends and future scenarios. Socio-Economic Planning Sciences, 29, 305-314.

Harwood H, Fountain D, Livermore G. (1998). The Economic Costs of Alcohol and Drug Abuse in the United States, 1992. Rockville: National Institutes on Drug Abuse.

Homer, J.B. (1993) A system dynamics model for cocaine prevalence estimation and trend projection. Journal of Drug Issues, 23, 251-279.

Kandel, D.B. (2002). Stages and Pathways of Drug Involvement: Examining the Gateway Hypothesis. New York: Cambridge University Press.

Levy, D.T., F. Chaloupka, J. Gitchell, D. Mendez, and K.E. Warner (2002). The use of simulation models for the surveillance, justification and understanding of tobacco control policies., Health Care Management Science, 5, 113-120.

Levy, D.T. and K. Friend (2002). Examining the effects of tobacco treatment policies on smoking rates and smoking related deaths using the SimSmoke computer simulation model. Tobacco Control, 11, 47-54.

Mendez, D. and K.E. Warner (2000). Smoking prevalence in 2010: Why the healthy people goal is unattainable. American Journal of Public Health, 90, 401-403.

Moore, T.J. (2006). The Social Costs of Illicit Drugs in Australia, Report for the Australian Federal Police. Fitzroy: Turning Point Alcohol and Drug Centre.

Ritter, A.J., G. Bammer, M. Hamilton, and L. Mazerolle (2007). Effective Drug Policy: A New Approach Demonstrated in the Drug Policy Modeling Program. Drug and Alcohol Review, 26(3): 265-271.

Rossi, C. (2001). A mover-stayer type model for epidemics of problematic drug use. Bulletin on Narcotics, 53, 39-64.

Rossi C. (2004). Operational models for epidemic of problematic drug use: the MoverStayer approach to heterogeneity. Socio-Economic Planning Sciences, 38, 73-90.

Weatherburn, D., C. Jones, K. Freeman, and T. Makkai (2003). Supply control and harm reduction: Lessons from the Australian heroin 'drought'. Addiction, 98, 83-91. 
Figure 1. Diagram of Drug Use Model's Five States and Associated Transitions

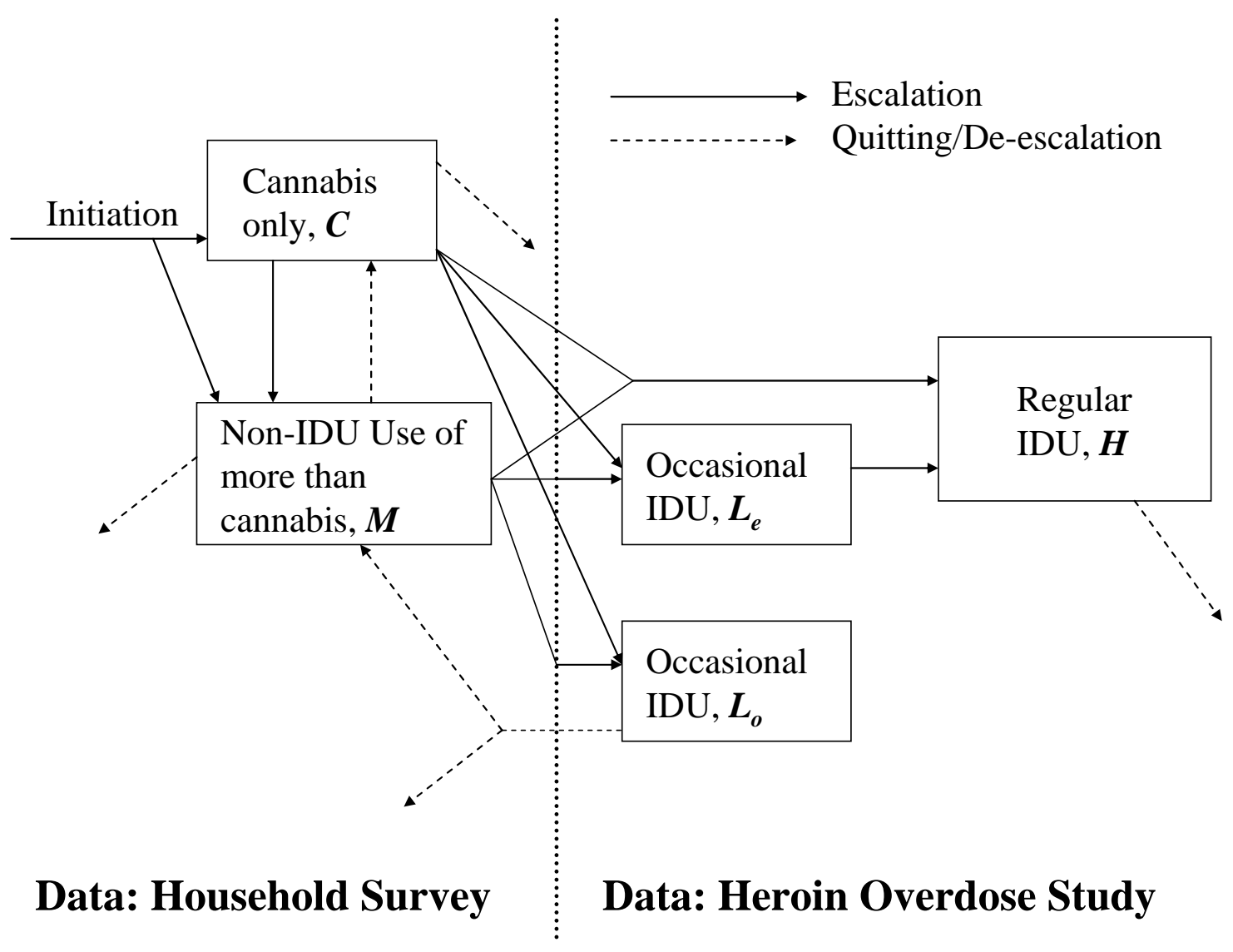


Figure 2: US Drug Initiation Over Time As a Percentage of the Pre-1990s Peak; Time Scaled Shifted to Align the Periods of Rapid Growth in Initiation

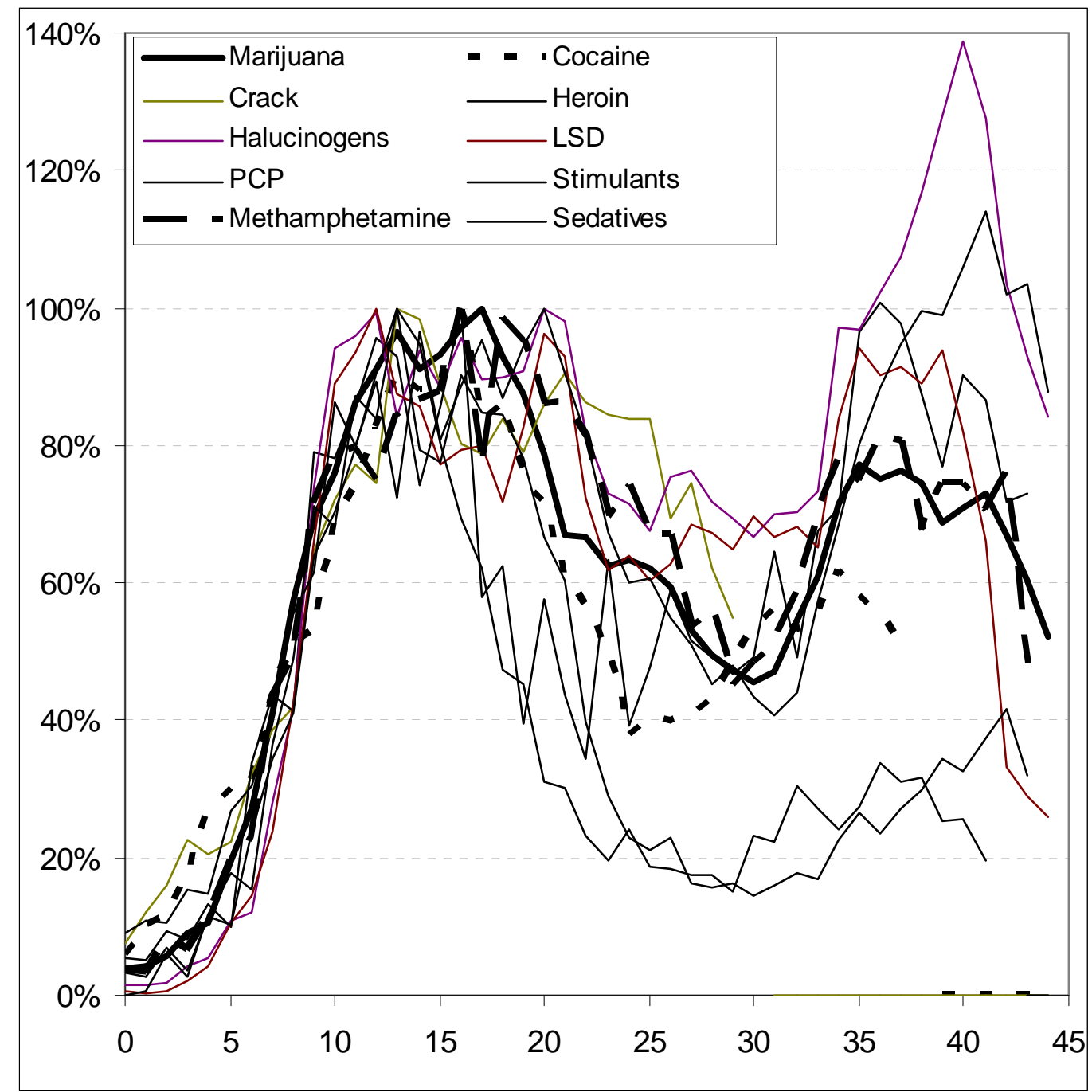


Figure 3: A Simple Trigonometric Model of Damped Oscillation Plotted Alongside Historical Trends in US Initiation of Marijuana and the Average of Nine Other

Drugs, Shifted to Align the Periods of Rapid Increase in Initiation

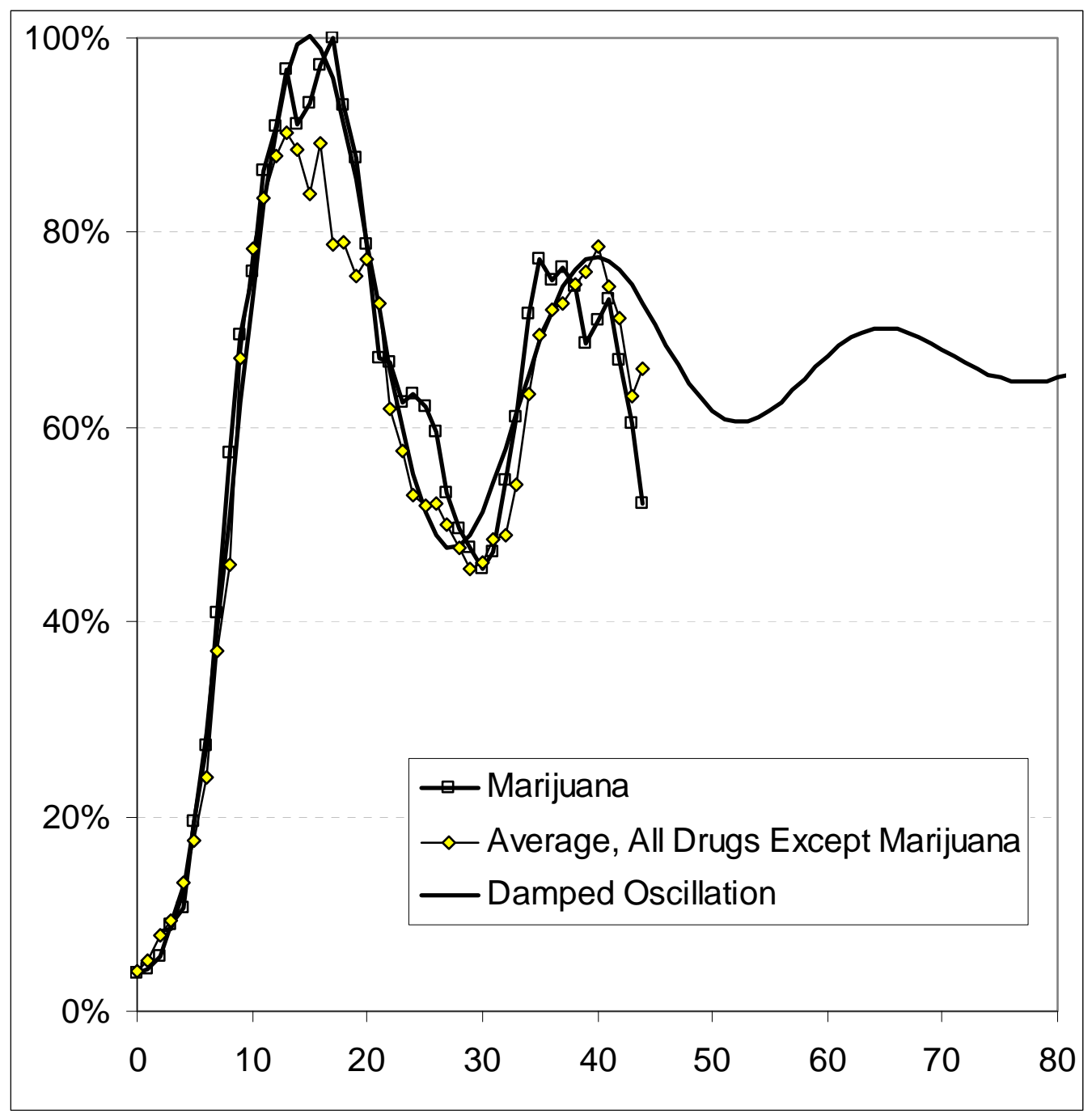


Figure 4. Damped Oscillation in Initiation Produces Modest Fluctuations in Prevalence and Steady Growth in Drug-Related Social Cost

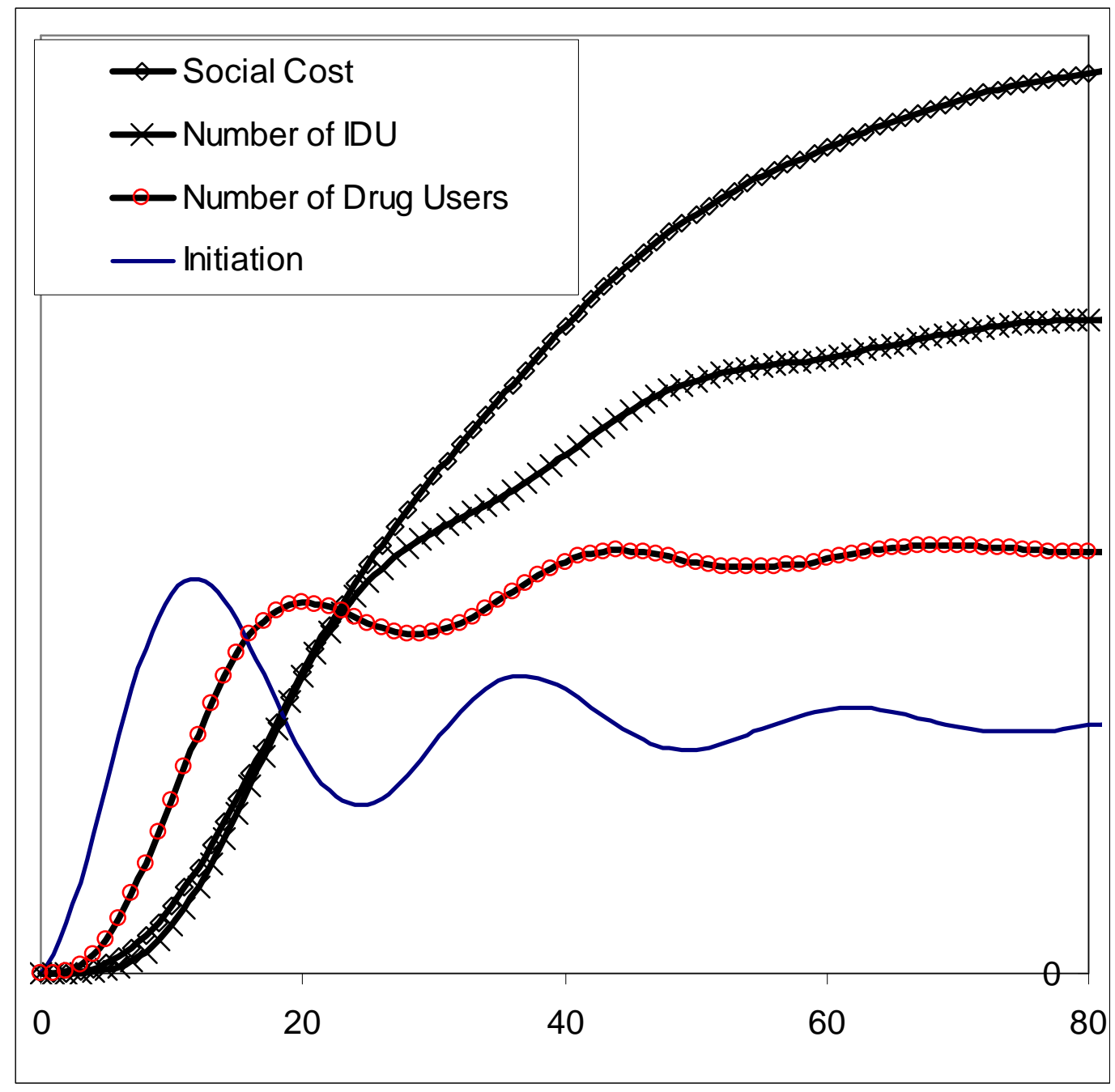


Figure 5. Even Un-Damped Oscillation in Initiation Produces Only Modest Fluctuations in Drug-Related Social Cost

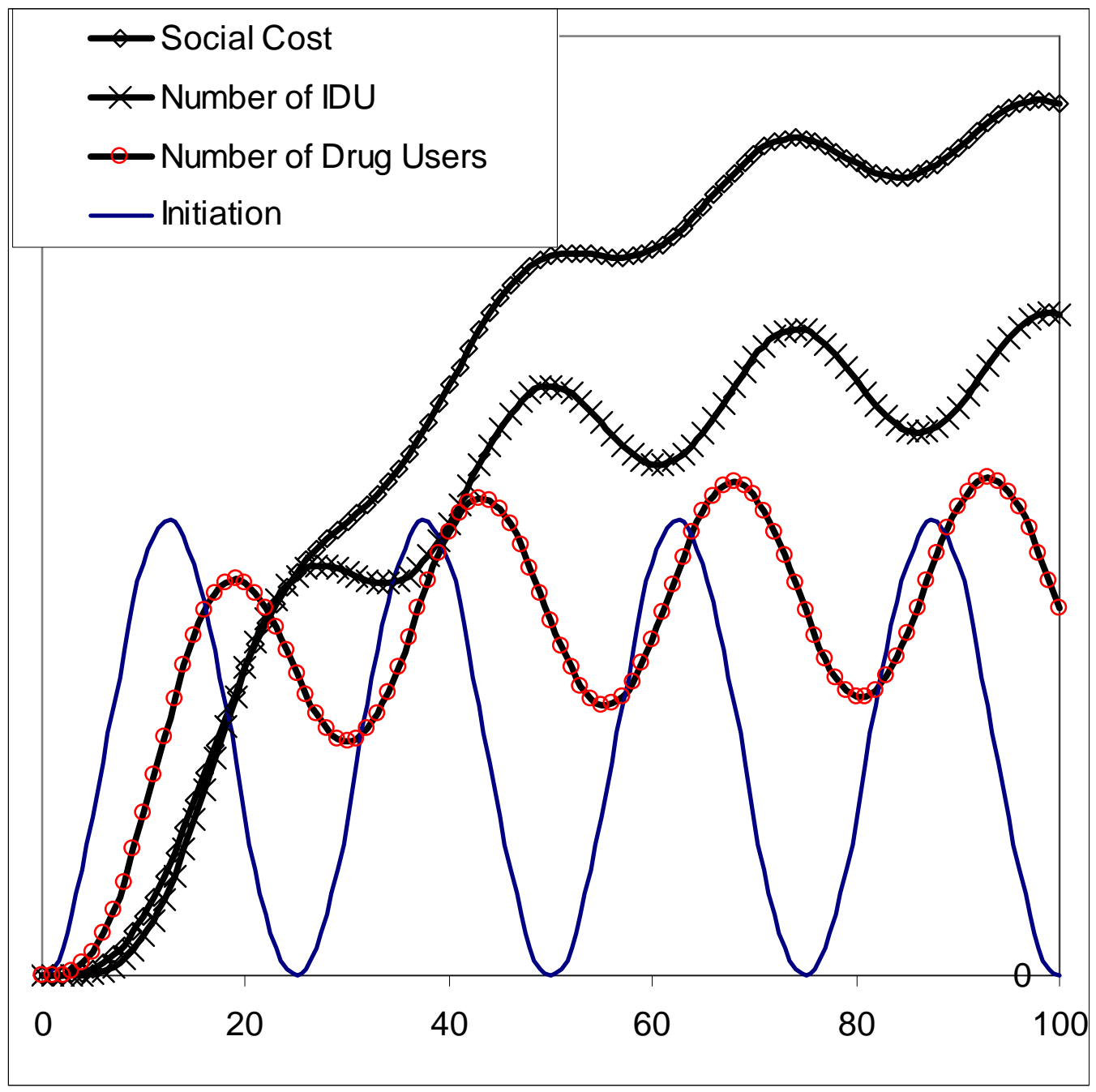


Figure 6. Effects of a Permanent 50\% Reduction in Initiation Starting in Year 13 and Relative to the Figure 4 Base Case

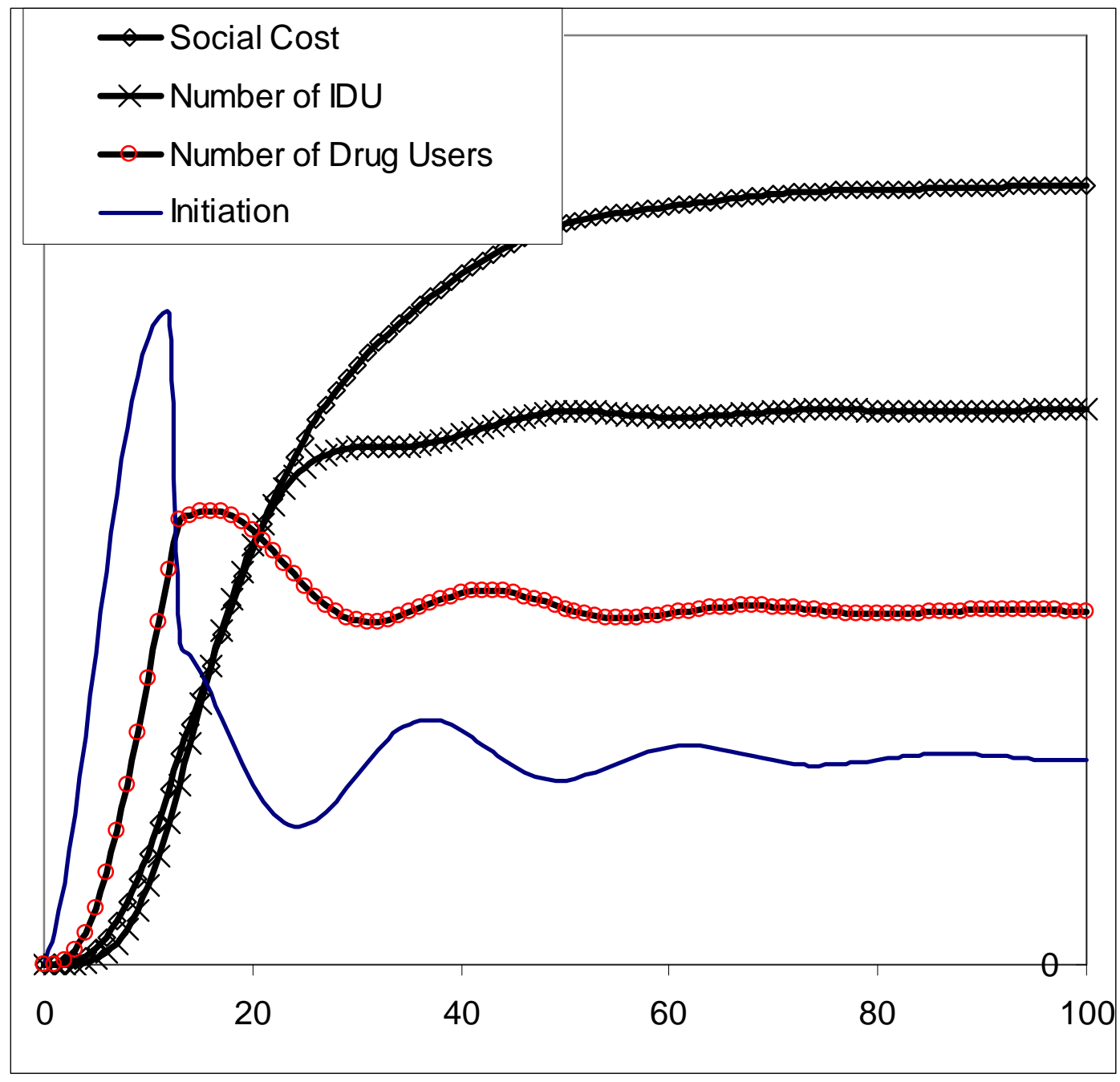


Figure 7. Halving Initiation for Two Years Creates Only a Modest Ripple in Future Drug-Related Social Costs, Even When a Perfect Counter-Factual Is Available

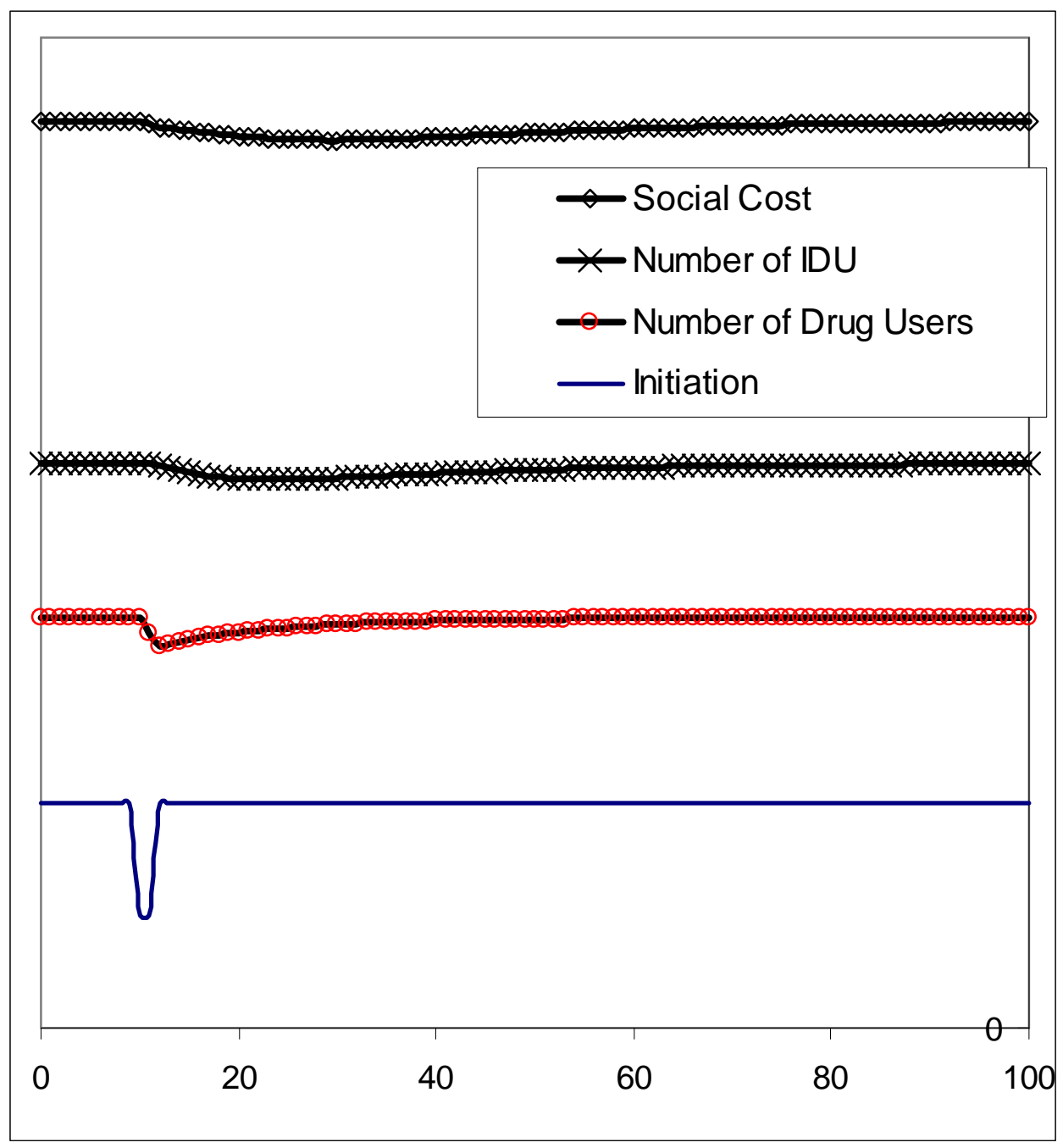

